\title{
Integration of Grey Model and Fuzzy system to Estimate the Obstacle Avoidance and Path Planning Robot
}

\author{
Tatsuyuki Nezu \\ Department of Chemical and Materials Engineering National Chin-Yi University of Technology \\ No.57,Sec.2, Zhong shanRd., Taiping Dist., Taichung 41170, Taiwan, ROC \\ E-mail: kelvinyu@ncut.edu.tw
}

\begin{abstract}
Keywords: laser range finder, obstacle avoidance, Segway, Fuzzy system, Grey system Abstract. The objective of this thesis is to make an obstacle avoidance and path planning robot by grey system theory. The robot is able to avoid dynamic and static obstacle automatically. The center position is defined by the motor encoders of the Segway RMP 50. The position sensor is a laser ranger finder in this system. It is installed on the top of the Segway to find out the environmental information for the grey obstacle avoidance controller. The robot is able to make an obstacle avoidance automatically.
\end{abstract}

\section{Introduction}

The purpose of this study is to use a laser range finder to scan the external environment and provide correct reference information to long-distance manipulation in unknown environment. The obstacle avoidance system, which is established through environmental information scanned by laser range finder, controls the robot to smoothly avoid dynamic and static obstacles, the fastest to reach the target location given by the host, and the successful completion of the task of autonomous walking. The coordinates of the starting point and the target point are preset in this study, environment information is scanned by using a laser range finder, and fuzzy walking controller is to determine obstacles in the walking process. When there is obstacle, the grey obstacle avoidance controller controls the robot to avoid obstacles, when no obstacle, the robot is to be continued the walking task, if not reach the target position, the robot return to fuzzy autonomous walking controller, walk to the target position and then stop ${ }^{[1-11]}$.

\section{Basic Theories}

\section{Grey system theory}

Grey system theory was pioneered by Deng Julong in 1982. Under the circumstance of the ambiguous system model and the incomplete information, the system is analyzed and understood by model constructing, relational analysis, prediction and decision. Grey theory is developed with the main information, control viewpoint combined with mathematical method. Up to now, grey system theory has been successfully applied to medicine, science, industry application and ecological assessment (Wu Hanxiong Deng Julong, Wenkun Li, 1998). Grey theory is characterized by a small amount of data to model, and its characteristics are as follows:

1. Grey dynamic model, as little as four samples.

2. Grey relational analysis model, each sequence is as little as three samples.

3. Grey Situation Decision, each goal is as little as three samples.

Grey Situation Decision Making: on a particular event, different game leads to different effect, and combined game with model to make the decision (Wukun Li, Zhao Zhongxian, 1998) ${ }^{[12-15]}$.

\section{Grey Situation Decision Making}

The main idea of Grey Situation Decision Making is as follows: event A occurs, game B to deal with A, which is the situation, and in many decisions of dealing with the same event, the decision-making is to pick the best game to deal with the occurred event, while the evaluation index of situation effect (Game effect) is called the decision target (target). 
Definition 1: $a_{i}$ as event, $b_{j}$ as game, the binary combination of $a_{i}$ with $b_{j}$ is called the situation, denoted as $S_{i j}$.

If $\mathrm{M}$ satisfied the conditions:
(a).M( $\left.u_{i j}^{p}\right)=r_{i j}^{p} e r^{p} \rightarrow r_{i j}^{p} \in[0,1]$
(b). $r_{i j}^{p} \in X^{+}$

\section{Grey obstacle avoidance}

The regional laser is divided into two districts, range 1 and range 2 in the thesis, which are $90^{\circ}$ sector. The actual sensing distance LR (Laser Radius) is set to $90 \mathrm{~cm}$ (Figure 1), according to the dodge of bodywork. When the object is too close to the body and enters into the preset radius, the system is changed to grey obstacle avoidance controller. When the system is in the state of autonomous walking, it uninterrupted detects the emergence of obstacles. When there is an obstacle, the system will switch to grey obstacle avoidance. Deviating from obstructions and leaving the detection range, the system will automatically switch to fuzzy walking and resume to the scheduled target coordinates.

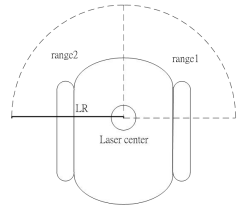

Fig. 1. Obstacle Avoidance laser regional schematic drawing.

\section{Grey obstacle avoidance controller}

Grey obstacle avoidance controller is started when encountered obstacles. Judged by LMS-100 regional laser, the number of obstacle points scanned by range 2 is greater than the points scanned by range1 (Figure 2), the controller is changed to the mode of left obstacle avoidance, otherwise, the controller is changed to the mode of right obstacle avoidance (Figure 3).

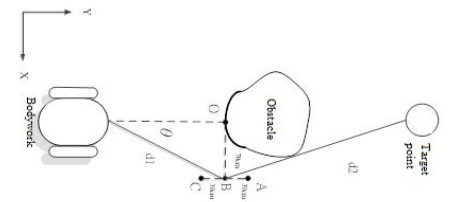

Fig. 2. Left obstacle avoidance mode dodge point diagram.

Table 1.the collection sample of grey situation (distance between target and bodywork is $250 \mathrm{~cm}$ ).

\begin{tabular}{cccc}
\hline $\begin{array}{l}\text { Virtual } \\
\text { dodgepoint }\end{array}$ & $\begin{array}{l}\text { Distance of the point with the } \\
\text { body } 1(\mathrm{~cm})\end{array}$ & Distance of point andtarget $\mathrm{d} 2(\mathrm{~cm})$ & $\begin{array}{l}\text { The angle of point with } \\
\text { obstructions and body } \theta \\
\text { (degree) }\end{array}$ \\
\hline $\mathrm{A}$ & 138.92 & 147.64 & 30.25 \\
\hline $\mathrm{B}$ & 114.01 & 174.64 & 37.87 \\
\hline $\mathrm{C}$ & 92.19 & 202.48 & 49.39 \\
\hline
\end{tabular}

The scanned and calculated data by laser are list in the Table 1 (from Fig 2 and 3), the effect measure of distance between the point and body is set to definite purposed measured equation (3), the effect measure of distance between the point and target is set to smaller-the-better measured equation (2), the effect measure of angle of points with obstacles and body is set to nominal-the-best equation (3), then set the weight to equal, elect the maximum value of effect measure by the means of calculation, and according to the best of situation, control the robot to reach the dodge point and obstacle avoidance (dodge points).

\section{Experimental Design of Grey System Theory}

\section{Laboratory equipment}

Segway RMP 50 is used as the robot mobile platform in this paper, and the original mobile platform is not matched the load bearing platform to carry the equipment, so it is necessary to design load bearing platform to carry all the equipment. The designed platform is the size of $40 \mathrm{~cm}$, width of $20 \mathrm{~cm}$, the top of load bearing platform is the diameter of $50 \mathrm{~cm}$ (Figure 4). On this platform, it can be equipped 
notebook computers, video cameras, sensors and other detection instruments, which is used in the end of control. The material of carrying platform is aluminum alloy ( $8 \mathrm{~mm}$ thick), not rusty, light weight, and not easily deformed. The top of load bearing disc is drilled round holes to device various instruments (Figure 5).

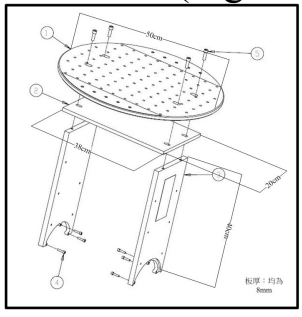

Fig. 4. Bearing platform operation drawing.

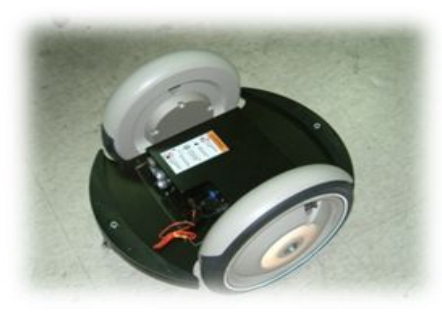

(a)RMP50Prototype appearance

Fig. 5. RMP 5 Bodywork.

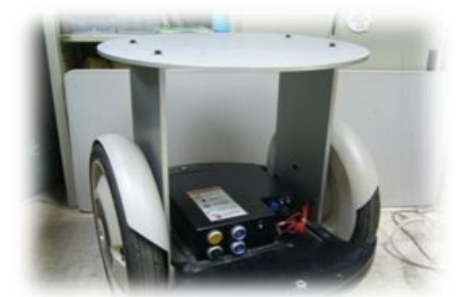

(b)The installation of the platform after the appearance

The host of RMP 50 is external Controlling PC arithmetic unit. The data sequence of 18 byte is transmitted through the USB of PC to CU_A and CU_B of RMP50, which to control the motor of RMP. In the data sequence, 9 Byte to 16 Byte are important information of robot (Table 1), including the return value of the RMP motor encoder and the command area of forward and back. The robot state will be able to know from the information, using conversion Table 2, the robot state can be converted into geodetic coordinates and angle, and the state of robot is more clearly to know.

\begin{tabular}{|c|c|c|}
\hline Byte & Value & Contents \\
\hline 0 & OxF0 & USB Message Header (Start Byte) \\
\hline \multirow[t]{2}{*}{1} & & USB Command Identifier \\
\hline & $0 \times 55$ & CAN message \\
\hline \multirow[t]{3}{*}{2} & & Command type \\
\hline & $0 \times 01$ & $\begin{array}{l}\text { CANA_DEV } \\
\text { Instructs UI to send message contents on CAN channel A (ignore on read) }\end{array}$ \\
\hline & $0 \times 05$ & $\begin{array}{l}\text { USB CMD_RESET } \\
\text { Sends a soft reset to the UI processor. }\end{array}$ \\
\hline 3 & $0 \times 00$ & Ignore on read, send as 0. \\
\hline 4 & $0 \times 00$ & Ignore on read, send as 0. \\
\hline 5 & $0 \times 00$ & Ignore on read, send as 0 . \\
\hline 6 & & CAN Message Header (high byte) \\
\hline 7 & & CAN Message Header (low byte) \\
\hline 8 & $0 \times 00$ & Ignore on read, send as 0 . \\
\hline 9 & & CAN Message Byte1 \\
\hline
\end{tabular}

\begin{tabular}{|l|l|l|}
\hline 10 & CAN Message Byte 2 \\
\hline 11 & CAN Message Byte 3 \\
\hline 12 & CAN Message Byte 4 \\
\hline 13 & CAN Message Byte 5 \\
\hline 14 & CAN Message Byte 6 \\
\hline 15 & CAN Message Byte 7 \\
\hline 16 & CAN Message Byte 8 \\
\hline 17 & $\begin{array}{l}\text { USB Message Checksum (8 bit one's complement) } \\
\text { See 'Computing Checksum' below for details. }\end{array}$ \\
\hline
\end{tabular}

Table 2. USB data sequence. 


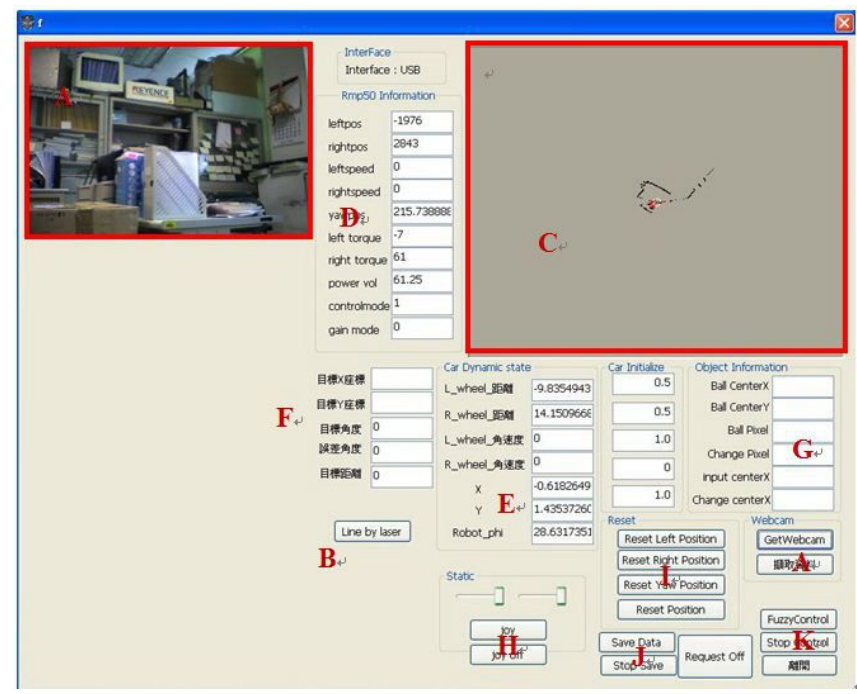

Fig. 6. bodywork man-machine interface.

\section{Bodywork entity man-machine interface}

The body can actually achieve the designed features using Visual $\mathrm{C}++\mathrm{MFC}$ software program in this paper. The functional software designed for this paper is shown in Figure 6, block A is vision system of robot, the function in this block contains the image processing, block B is to get environmental data to link robot with LMS-100, block C is built environmental data for LMS-100 scanning. The black spots in the figure are the point of laser scanning, this interface can detect whether there are obstacles near the robot.

\section{Experimental results}

In the scene of fixed obstacles, robot independently and straight travel to any specified location. Obstacle B is added in the path, and the error input verify the feasibility of the actual system. The top view of robot walking in 2D plane is described in Figure 7, the arrow is the walking direction for the robot. (1) is the starting position of the robot in Figure 7, corresponding to Figure 8 (1). (16) is the target point of the robot in Figure 7, corresponding to Figure 8 (16). In Figure 8, the block of black box is the end position [0,450], for the robot, the block of black round is starting position [0, 0], which is the example of a robot autonomous walking forward $450 \mathrm{~cm}$. Obstacle A and obstacle B are added in the predicted travel path to block the walking of robot, in addition to fuzzy controller, the grey obstacle avoidance controller is used to avoid obstacle. The system uninterruptedly detect the distance between the robot and the obstacle less than the designed critical value $(90 \mathrm{~cm})$, when the distance is smaller than the critical value, the system will immediately switch to the mode of grey obstacle avoidance control, instead of the mode of autonomous walking control, and the robot is able to avoid obstacle automatically. For the above, the continued function of autonomous walking can avoid this obstacle. When the robot is away from the obstacle, the system will immediately switch to the mode of autonomous walking control once again. The objectives can be achieved to avoid obstacles and reach the specified target location through the mode of switching. Through the exploded view of the present example, it can be found that the robot effectively avoid obstacle A and obstacle B. The robot is autonomous walking from the left to the right of $450 \mathrm{~cm}$ in the exploded view, Figure 8 (1) is the initial state of the robot, whose task is to dodge stationary obstacles and reach to the target position. Figure 8 (2) is the target position of the robot, straight line distance between the robot and the target position is $450 \mathrm{~cm}$, Figure 8 (3) is the relative position of the robot starting point and the target location, Figure 8 (4) shows the forward autonomous walking of robot, which is closed to the fixed obstacle A towards the target position. Due to the distance between the robot and obstacle less than the critical value, the control mode is switched to the mode of grey obstacle avoidance control. In Figure 8 (5), the robot has been switched into the mode of grey obstacle avoidance control and the mode of left obstacle avoidance judged by more obstacle point in the left. Through the program of 
grey decision-making and the most suitable dodge point, the robot executes right turning to dodge fixed obstacle A. Figure 8 (6) shows that the robot has reached the dodge point (the point B in Figure $3)$.

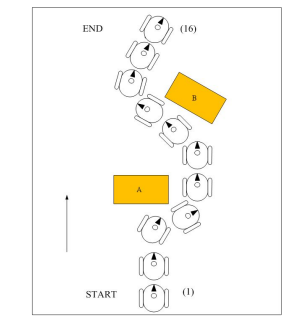

Fig. 7. Avoiding obstacles continuous movement drawing.

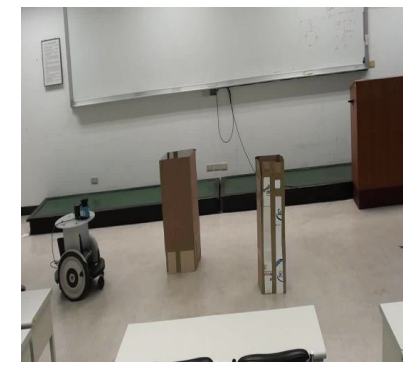

Fig. 8. Exploded view of the autonomous walking robot.

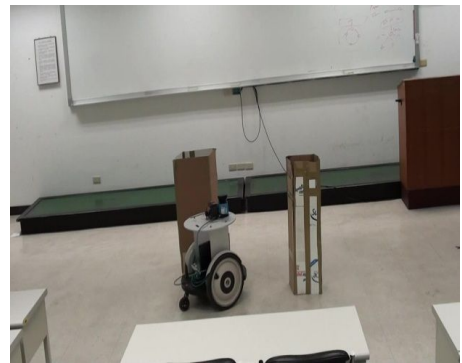

\section{Conclusion}

In the paper, the wheeled mobile robot is point-to-point autonomous walking. The fixed static and instant random dynamic obstacle is in the path, obstacle avoidance controller is designed by the means of grey system theory. The input data are the distance between three dodge point and the vehicle body, the distance between three dodge point and the target, as well as the angle of the obstacle and the vehicle body.By means of the grey decision analysis, an optimum dodge point is obtained. The output of two angular velocity values of robot is controlled by the fuzzy system controller. The experimental results show the intelligent robot works very well and the control system is feasible.

\section{References}

[1] Li Changjun., Zhao Jinhou.(2004). Optimized Operation of Gas Transmission Pipelines, Natural Gas Industry[J], 25(10), 106-109.

[2] Li Changjun (2008). Natural Gas Pipeline Transmission[M]. Beijing, Petroleum Industry Press

[3] Deng Julong (1990). A Course on Grey System Theory[M]. Wuhan, Huazhong Univ.of Sci. \& Tech. Press

[4] Wang Yuchun(1993). A New Method of Optimally Designing Transmission Line, Natural Gas Industry[J], 13(6), 64-69.

[5] Jia Wenlong, Li Changjun(2009). Comprehensive Evaluation on Regulation Schemes of Gas Transmission Pipelines[C]. ASCE International Conference on Pipeline and Trenchless Technology, 2009,581-590.

[6] Liang Guangchuan., Zheng Yunping (2004). Grey Correlation Analysis of Optimized Design for Underground Gas Storage. Natural Gas Industry]y[J], 24(9),142-144.

[7] Yang Dewei, Zhao Xiaoming, Zhang Aiyan (2007). Application of grey relation analysis method to efficiency evaluation of stations and tank farms in oil and gas gathering and transportation system. Journal of China University of Petroleum [J], 31( 4),98-104.

[8] Saaty T. L.(1980). The Analytic Hierarchy Process [M]. New York: McGraw-Hill.

[9] Bhushan Navneet, Kanwal Rai (2004). Strategic Decision Making: Apply the Strategic Decision Making: Applying the Analytic Hierarchy Process [M]. London: Springer-Verlag.

[10]Liang Liang, Sheng Zhaohan(1989). An improved Analytic Hierarchy Process, System Engineering[J], 7(3) 